\title{
CONTINUITY AND HOMEOMORPHISM GROUPS
}

\author{
ROBERT ELLIS
}

Let $X$ be a $T_{2}$-space, let $\Phi$ be a group of homeomorphisms of $X$ onto $X$, let $\Pi: X \times \Phi \rightarrow X$ be the map such that $(x, \phi) \Pi=x \phi$ $(x \in X, \phi \in \Phi)$, and for each $x \in X$ let $\Pi_{x}: \Phi \rightarrow X$ be the map such that $\phi \Pi_{x}=(x, \phi) \Pi=x \phi$.

Lemma 1. Let $X$ be a regular Baire space (that is, every countable intersection of everywhere dense open sets is everywhere dense), let $\mathcal{T}$ be a first countable topology on $\Phi$ such that each $\Pi_{x}(x \in X)$ is continuous, let $x_{0} \in X$, let $\phi \in \Phi$, and let $U$ be a neighborhood of $x_{0} \phi$. Then there exist a neighborhood $N$ of $\phi$ and a nonvacuous open subset $V$ of $X$ such that $(V \times N) \Pi \subset U$.

Proof. There exists an open neighborhood $W$ of $x_{0} \phi$ such that $\operatorname{cls} W \subset U$, where cls $W$ denotes the closure of $W$. Let $\left[N_{k} \mid k=1,2, \cdots\right]$ be a neighborhood base at $\phi$. Let $C_{k}=[x \mid x \in X$ and $N_{k} \Pi_{x} \subset$ cls $\left.W\right]$. Then $C_{k}=\bigcap_{\phi \in N_{k}}(\operatorname{cls} W) \phi^{-1}$ which is closed. Moreover $W \phi^{-1} \subset \bigcup_{k=1}^{\infty} C_{k}$ as follows. Let $x \in W \phi^{-1}$. Then $x \phi \in W$, i.e. $\phi \Pi_{x} \in W$. Thus there exists $k$ such that $N_{k} \Pi_{k} \subset W \subset$ cls $W$. Hence $x \in C_{k}$. Now from $W \phi^{-1} \subset U_{k-1}^{\infty} C_{k}$ it follows that int $\cup_{k-1}^{\infty} C_{k} \neq \varnothing$ and there exists $k$ such that int $C_{k} \neq \varnothing$. Let $V=$ int $C_{k}$ and $N=N_{k}$. Then $(V \times N) \Pi \subset U$. The proof is completed.

The group $\Phi$ is rigid provided that if $\mathcal{F}$ is a filter base on $\Phi$ and if $x, y, z \in X$ are such that $x \mathcal{F} \rightarrow z$ and $y \mathcal{F} \rightarrow z$, then $x=y$. Rigidity may be characterized as follows. The group $\Phi$ is rigid if and only if given $x, y, z \in X$ such that $x \neq y$ there exists a neighborhood $U$ of $z$ such that if $\phi \in \Phi$ and $x \phi \in U$, then $y \phi \notin U$. Thus the notion of rigidity used here is equivalent to the one attributed to Hilbert by Zippin [3]. For emphasis one might refer to the above concept as pointwise rigidity in contrast to uniform rigidity which might be defined as follows, provided that $X$ is a uniform space. The group $\Phi$ is uniformly rigid provided that if $x, y \in X$ such that $x \neq y$, then there exists an index $\alpha$ of $X$ such that $x \phi \notin y \phi \alpha$ for all $\phi \in \Phi$. It can be shown that in case $X$ is compact the notions are equivalent.

THEOREM 1. Let $X$ be locally compact, let $\Phi$ be abelian and rigid, let $\tau$ be a first countable topology on $\Phi$ which makes each $\Pi_{x}(x \in X)$ continuous, and let $x_{0} \in X$ be such that cls $\left(x_{0} \Phi\right)=X$. Then $\Pi$ is continuous at $\left(x_{0}, \phi\right)$ for all $\phi \in \Phi$.

Presented to the Society, April 25, 1953; received by the editors March 12, 1953. 
Proof. Let $\phi \in \Phi$, let $\mathcal{A}$ be a countable neighborhood base at $\phi$, and let $\mathcal{F}$ be a filter base on $X$ such that $\mathcal{F} \rightarrow x_{0}$. It is required to show that $(\mathcal{F} \times \mathcal{A}) \Pi \rightarrow x_{0} \phi$. Let $B$ be the neighborhood base of $x_{0} \phi$ consisting of compact neighborhoods only and let $U \in B$. By Lemma 1 there exist $A \in \mathcal{A}$ and a nonvacuous open subset $W$ of $X$ such that $(W \times A) \Pi \subset U$. Since cls $\left(x_{0} \Phi\right)=X$, there exists $\psi_{U} \in \Phi$ such that $W \psi_{U}$ is a neighborhood of $x_{0}$. The group $\Phi$ being abelian, we conclude that $\left(W \psi_{U} \times A\right) \Pi \subset U \psi_{U}$. There exists $F \in \mathcal{F}$ such that $F \subset W \psi_{U}$ and hence $(F \times A) \Pi \subset U \psi_{U}$. Since $U$ is compact, so is $U \psi_{U}$ and hence $(\mathcal{F} \times \mathcal{A}) \Pi$ has an adherent point in $U \psi_{U}$. To show $(\mathcal{F} \times \mathcal{A}) \Pi \rightarrow x_{0} \phi$ it suffices to show that the only possible adherent point of $(\mathcal{F} \times \mathcal{A}) \Pi$ is $x_{0} \phi$. Let $y$ be adherent to $(\mathcal{F} \times \mathcal{A}) \Pi$. Then $y \in U \psi_{U}$ and $y \psi_{U}^{-1} \in U$. Set $G_{U}$ $=\left[\psi_{V}^{-1} \mid V \in \mathbb{B}\right.$ and $\left.V \subset U\right]$. Then $G=\left[G_{U} \mid U \in B\right]$ is a filter base on $\Phi$ such that $y G \rightarrow x_{0} \phi$. But the relation $\left(W \psi_{U} \times A\right) \Pi \subset U \psi_{U}$ shows that $x_{0} \phi \in U \psi_{U}$ and consequently that $x_{0} \phi G \rightarrow x_{0} \phi$. Hence by the rigidity of $\Phi$, we conclude that $y=x_{0}$. The proof is completed.

CoROLlaRY 1. Under the same assumptions as in Theorem 1 we conclude that $\Pi$ is continuous on $x_{0} \Phi \times \Phi$.

Corollary 2. Let $X$ be a first countable locally compact $T_{2}$-space with an abelian group structure such that multiplication is unilaterally continuous. Then multiplication is bilaterally continuous.

Leмma 2. Let $X$ be a $T_{2}$-space with a group structure such that multiplication is continuous and let $H$ be a compact subset of $X$. Then $H^{-1}$ is closed.

Proof. Let $\mathcal{F}$ be a filter base on $H^{-1}$ such that $\mathcal{F} \rightarrow x \in X$. It is enough to show that $x \in H^{-1}$. Let $U$ be an ultra-filter on $H^{-1}$ such that $\mho \supset \mathcal{F}$. Then $U \rightarrow x$ and $V^{-1}$ is an ultra-filter on $H$. Consequently $U^{-1} \rightarrow y \in H$ and $U U^{-1} \rightarrow x y$ which must be the identity since $X$ is $T_{2}$. Thus $x=y^{-1} \in H^{-1}$. The proof is completed.

THEOREM 2. Let $X$ be a separable first countable locally compact $T_{2}$-space with an abelian group structure such that multiplication is unilaterally continuous. Then $X$ is a topological group.

Proof. By Corollary 2 it suffices to show that the map $x \rightarrow x^{-1}$ is continuous. To do this it is sufficient to show that if $V$ is a neighborhood of the identity $e$, then int $V^{-1} \neq \varnothing$, as follows. Let $\left(x_{n} \mid n=1, \cdots\right)$ be a sequence such that $x_{n} \rightarrow e$ and let $U$ be a compact neighborhood of $e$. Since int $U^{-1} \neq \varnothing$ by assumption, there exist $y \in X$ and an integer $N$ such that $n>N$ implies $x_{n} \in U^{-1} y^{-1}$ or $x_{n}^{-1} \in y U$ for $n>N$. Since $U$ is compact, we have $x_{n} \rightarrow x_{0}$ for some 
$x_{0} \in X$. But multiplication being bilaterally continuous we have $x_{0}=e$. Consequently we need only show that int $V^{-1} \neq \varnothing$ for any compact neighborhood $V$ of $e$. Since $X$ is separable there exists a countable subset $E$ of $X$ such that cls $E=X$. Moreover $E V^{-1}=X$. But by Lemma $2, V^{-1}$ is closed since $V$ is compact. Consequently int $V^{-1} \neq \varnothing$ since $X$ is a Baire space. The proof is completed.

COROLlaRY 3. Under the same assumptions as in Theorem 2, we have that $X$ is metrizable.

Lemma 2 and Theorem 2 are closely related to a result of Montgomery's [2] which dealt with similar questions in complete metric spaces. An interesting question along these lines is whether the hypothesis of first countability can be removed in Lemma 2.

For the remainder of the paper we suppose that $X$ is a separated uniform space and that each $\Pi_{x}(x \in X)$ is one-to-one.

The proofs of Lemmas 3 and 4 are straightforward and will therefore be omitted.

Lemma 3. There exists a (necessarily unique) topology $\mathcal{T}$ of $\Phi$ which makes each $\Pi_{x}(x \in X)$ homeomorphic if and only if each $\Pi_{x}^{-1} \Pi_{y}: x \Phi$ $\rightarrow y \Phi(x, y \in X)$ is homeomorphic. If the topology $\mathcal{T}$ exists, then $\mathcal{T}$ is the point-open topology on $\Phi$.

Lemma 4. Let $X$ be a minimal orbit-closure under $\Phi$, that is, let $x \in X$ imply cls $(x \Phi)=X$. Then $\Phi$ is equicontinuous if and only if the restriction to $x \Phi$ of $\Phi$ is equicontinuous for each $x \in X$.

The group $\Phi$ is said to be finitely controlled provided that for every $\phi \in \Phi$ and every index $\alpha$ of $X$ there exist an index $\beta$ of $X$ and a finite subset $E$ of $X$ such that if $\psi \in \Phi$ and if $(x \psi, x \phi) \in \beta$ for all $x \in E$, then $(x \psi, x \phi) \in \alpha$ for all $x \in X$. In other words, $\Phi$ is finitely controlled if and only if the topology on $\Phi$ of pointwise convergence coincides with the topology on $\Phi$ of uniform convergence. Notice that no mention is made of the corresponding uniformities on $\Phi$, in this characterization.

LeMMA 5. Let $X$ be a minimal orbit-closure under $\Phi$ and let each $\Pi_{x}^{-1} \Pi_{v}(x, y \in X)$ be homeomorphic. Then $\Phi$ is rigid.

Proof. Let $\mathcal{F}$ be a filter base on $\Phi$ and let $x, y, z \in X$ be such that $x \mathcal{F} \rightarrow z$ and $y \mathcal{F} \rightarrow z$. Since cls $(z \Phi)=X$, there exists a filter base $G$ on $\Phi$ such that $x G \rightarrow x$ and $y G \rightarrow x$. Therefore $y G=(x G) \Pi_{x}^{-1} \Pi_{y} \rightarrow x \Pi_{x}^{-1} \Pi_{y}$ $=y$ and $x=y$. The proof is completed.

THEOREM 3. Let $X$ be a minimal orbit-closure under $\Phi$, let $\Phi$ be abelian and finitely controlled, and let each $\Pi_{x}^{-1} \Pi_{y}(x, y \in X)$ be homeomorphic. 
Then $\Phi$ is equicontinuous.

Proof. Let $x \in X$. By Lemma 4 it suffices to show that the restriction to $x \Phi$ of $\Phi$ is equicontinuous. To this end let $\phi \in \Phi$ and let $\alpha$ be an index of $X$. We need to find a neighborhood $U$ of $x \phi$ such that ( $U \cap x \Phi) \psi \subset x \phi \psi \alpha$ for all $\psi \in \Phi$. Since $\Phi$ is finitely controlled, there exists an index $\beta$ of $X$ and a finite subset $E$ of $X$ such that if $\theta \in \Phi$ and if $(y \theta, y \phi) \in \beta$ for all $y \in E$, then $(y \theta, y \phi) \in \alpha$ for all $y \in X$. There exists a neighborhood $U$ of $x \phi$ such that $(U \cap x \Phi) \Pi_{x}^{-1} \Pi_{y} \subset y \phi \beta$ for $y \in E$. This is the required neighborhood as shown by the following. Let $\psi \in \Phi$ and let $z \in U \cap x \Phi$. Then $z=x \theta$ for some $\theta \in \Phi$. Since $(U \cap x \Phi) \Pi_{x}^{-1} \Pi_{y} \subset y \phi \beta$ for all $y \in E$, it follows that $y \theta=z \Pi_{x}^{-1} \Pi_{y} \in y \phi \beta$ for all $y \in E$. Therefore $y \theta \in y \phi \alpha$ for $y \in X$. Thus $z \psi=x \theta \psi=x \psi \theta \in x \psi \phi \alpha$ $=x \phi \psi \alpha$; i.e. $(U \cap x \Phi) \psi \subset x \phi \psi \alpha$. The proof is completed.

Theorem 4. Let $X$ be a minimal orbit-closure under $\Phi$, let $X$ be first countable compact, let $\Phi$ be abelian, and let each $\Pi_{x}^{-1} \Pi_{y}(x, y \in X)$ be homeomorphic. Then $\Phi$ is equicontinuous.

Proof. By Theorem 3 it suffices to show that $\Phi$ is finitely controlled. Since $X$ is compact this is equivalent to showing that $\Pi$ is continuous when $\Phi$ is provided with the point-open topology. By Lemma 3 the point-open topology on $\Phi$ is merely the inverse image topology induced on $\Phi$ by the map $\Pi_{x}$ where $x \in X$, and is consequently first countable. Lemma 5 shows that $\Phi$ is rigid, and hence by Theorem 1, $\Pi$ is continuous. The proof is completed. The theory of uniform spaces together with Theorem 4 shows that under the above assumptions $X$ is metrizable. It would be interesting to find out whether $X$ remains metrizable if the condition that $\Pi_{x}^{-1} \Pi_{y}(x, y \in X)$ be homeomorphic is deleted. Theorem 4 generalizes a result due to Gottschalk [1].

In connection with the concept of rigidity used in this paper one might mention the notion of regional rigidity. The group $\Phi$ is regionally rigid provided that if $x, y, z \in X$ such that $x \neq y$, then there exist neighborhoods $U, V, W$ of $x, y, z$ respectively such that if $\phi \in \Phi$ and $W \cap U \phi \neq \varnothing$ then $W \cap V \phi=\varnothing$. This is what Zippin refers to as Axiom $\mathrm{H}$. The latter part of the paper may be conveniently summarized in the following theorem.

TheORem 5. Let $X$ be a first countable compact $T_{2}$-space which is also a minimal orbit-closure under the abelian group $\Phi$. Then the following statements are pairwise equivalent.

1. The group $\Phi$ is regionally rigid.

2. The maps $\Pi_{x}^{-1} \Pi_{y}(x, y \in X)$ are homeomorphic. 
3. The group $\Phi$ is equicontinuous.

Proof. Statement 2 implies statement 3 by Theorem 4 . Now assume statement 3 holds. Let $x \in X$. Then since cls $(x \Phi)=X$, the point-open topology on $\Phi$ relative to $X$ coincides with the pointopen topology on $\Phi$ relative to $x \Phi$ which is merely the inverse image topology on $\Phi$ induced by the map $\Pi_{x}$. Thus statement 2 holds.

Now assume statement 1 . Let $x \in X$ and let $U$ be an open neighborhood of $x$. If $y \in U^{\prime}=X-U$, then there are neighborhoods $V_{y}$ of $x$ and $W_{y}$ of $y$ such that if $\phi \in \Phi$ and $V_{y} \cap V_{y} \phi \neq \varnothing$, then $V_{y} \cap W_{y} \phi=\varnothing$. Since $U^{\prime}$ is compact, there exists a finite subset $F$ of $U^{\prime}$ such that $\cup_{\nu \in F} W_{y} \supset U^{\prime}$. Let $V=\bigcap_{\nu \in F} V_{y}$. Then if $\phi \in \Phi$ and $V \phi \cap U \neq \varnothing$ then $V \phi \cap U^{\prime}=\varnothing$. Hence, because $X$ is a minimal orbit-closure, we may conclude statement 3 .

Finally, assume statement 3 . Let $x, y, z \in X$ such that $x \neq y$. There exists an index $\alpha$ of $X$ such that $(x, y) \notin \alpha$. Since $X$ is compact, $\Phi$ is uniformly equicontinuous. Hence there exists an index $\beta$ of $X$ such that $(u, v) \in \beta$ and $\phi \in \Phi$ imply $(u \phi, v \phi) \in \alpha$. Let $\gamma$ be a symmetric index of $X$ such that $\gamma^{4} \subset \beta$. Set $U=x \gamma, V=y \gamma$, and $W=z \gamma$. A simple computation shows that $U, V$, and $W$ have the property required in the definition of regional rigidity. The proof is completed.

\section{REFERENCES}

1. W. H. Gottschalk, Transitivity and equicontinuity, Bull. Amer. Math. Soc. vol. 54 (1948) pp. 982-984.

2. D. Montgomery, Continuity in topological groups, Bull. Amer. Math. Soc. vol. 42 (1936) pp. 879-882.

3. L. Zippin, Transformation groups, Lectures in Topology, The University of Michigan, Conference of 1940.

University of Pennsyluania 